\title{
On the Gauged Non-compact Spin System
}

\author{
Sung-Soo Kim円 and Phillial Oh[ \\ Department of Physics, Sung Kyun Kwan University, Suwon 440-746, Korea
}

\begin{abstract}
We examine classical and quantum aspects of the planar non-compact spin system coupled with Chern-Simons gauge field in the presence of background charge. We first define our classical spin system as non-relativistic non-linear sigma model in which the order parameter spin takes value in the non-compact manifold $\mathcal{M}=$ $S U(1,1) / U(1)$. Although the naive model does not allow any finite energy self dual solitons, it is shown that the gauged system admits static Bogomol'nyi solitons with finite energy whose rotationally symmetric soliton solutions are analyzed in detail. We also discuss the large spin limit in which the self-dual equation reduces to the well-known gauged nonlinear Schrödinger model or Abelian Higgs model, depending on the choice of the background charge term. Then, we perform quantization of the model. We find that the spin algebra satisfies anomalous commutation relations, and the system is a field theoretic realization of the anyons.
\end{abstract}

PACS codes: 11.10.Lm, 11.27.+d

\footnotetext{
${ }^{1}$ Electronic-mail: billy@newton.skku.ac.kr

${ }^{2}$ Present Address: Center for Theoretical Physics, M.I.T., Cambridge, MA 02139-4307
} 


\section{Introduction}

Recently, a nonrelativistic nonlinear sigma model (NLSM) defined on the target space of coadjoint orbits $G / H$ was proposed, whose Euler-Lagrange equation of motion yields the generalized continuous Heisenberg ferromagnet [1]. In this system, the dynamical variable is spin defined on coadjoint orbit whose Poisson bracket satisfies the classical $\mathcal{G}$ algebra. When the target spaces of coadjoint orbits are given by Hermitian symmetric spaces (HSS) [2] which are symmetric spaces equipped with complex structure, the generalized spin system becomes completely integrable in $1+1$ dimension. It was also discovered that incorporation of the Chern-Simons (CS) gauge field in $2+1$ dimension on the same target space produces a class of self-dual field theories. They admit Bogomol'nyi type self-dual equations whose energy is saturated by the topological charge [3]. A detailed numerical investigation in the compact $S U(2)$ case [4] showed a rich structure of rotationally symmetric self-dual CS solitons with finite energies.

Since the self-dual CS solitons attracted a upsurge of recent theoretical interest [5], it is worthwhile to give a more detailed investigation to the gauged spin system. In this paper, we carry out this in the case of CS gauged non-compact symmetry. We consider the simplest non-compact $G=S U(1,1)$ with the target space of the hyperboloid $\mathcal{M}=S U(1,1) / U(1)$ which is a well-known example of the noncompact HSS. The motivations for non-compact symmetry are two-fold. On the classical aspect, we are interested in the Bogomol'nyi self-dual solitons with finite energy. First of all, the non-compact symmetry in nonlinear sigma model was considered in a wide context before, in relation with dynamical generation of gauge bosons in supergravity theory [6], integrable non-linear Schrödinger equation in 1+1 dimension [7], Ernest equation in 2+1 dimension [8], and also in the context of condensed matter physics such as integer quantum Hall effect [9] and magnetic spin system [10]. The static self-dual Bogomol'nyi solitons with non-compact symmetry were studied only recently in [11] where it was shown that relativistic NLSM on $\mathcal{M}$ coupled with the CS gauge field admits rotationally symmetric soliton solutions. On the non-relativistic side, the result of Ref. [3] on self-dual CS theories in the arbitrary HSS implies that the non-compact spin system will admit the self-dual equations with Bogomol'nyi bound. But this does not guarantee the existence of finite positive semi-definite energy solitons. One of our main purpose is to survey the existence of static self-dual Bogomol'nyi solitons with finite energy in 
the gauged non-compact spin system.

The non-compact spin variables are defined on the target space $\mathcal{M}$. In contrast to the compact $S U(2)$ case on $S^{2}$ which is a typical example of circumventing the well-known no-go theorem in static solitons [12], and is supported by the finite energy topological lump solutions characterized by the integer winding, $\pi_{2}\left(S^{2}\right)=\mathbf{Z}$ [13], the ungauged non-compact spin system does not allow any static solitons with an integer winding because of $\pi_{2}(\mathcal{M})=0$. Besides, the self-dual equations have only singular solutions in which both the energy and topological charge diverge (see discussion following (8)). Coupling with other matter field improves the situation in that there exist exact solutions characterized by a finite topological charge, but they are singular and not localized soliton solutions [14. Incorporation of the gauge field drastically changes the situation. A detailed investigation of the gauged self-dual equations and their numerical solutions indeed reveals that the gauged non-compact spin system admits self-dual CS solitons with finite energy.

Another aspect of interest is that the system permits classical Holstein-Primakoff transformation [15 $(\psi \rightarrow \vartheta)$ which changes the symplectic one-form on $\mathcal{M}(\sim$ $\left.\frac{\psi^{*} \dot{\psi}-\dot{\psi}^{*} \psi}{1-|\psi|^{2}}\right)$ into the canonical structure $\left(\sim \vartheta^{*} \dot{\vartheta}\right)$. This transformation has an oneparameter dependence on the total spin and we find that in the large spin limit, the self-dual equations reduce to the well-known gauged nonlinear Schrödinger model of Jackiw and Pi [16], or Abelian Higgs model [17], depending on the choice of the background charge term.

The second is quantum aspect, especially concerned with realization of anyons [18]. It is well-known that the CS field produces the long-range interaction which is responsible for the existence of anyon. We find that the quantization of the gauged non-compact spin system also leads to the nonrelativistic field theory describing anyons, and in the large spin limit it again reduces to the gauged nonlinear Schrödinger model. Of course, these features are not something which belongs only to non-compact symmetry. The same analysis performed in this paper also indicates that the compact version of the model [4] also exhibits the anyon characteristic. But it is interesting to have these properties in the non-compact case also.

The paper is organized as follows: In Section 2, we first describe the ungauged non-compact spin system and set the framework for further discussion. Then, we couple with the CS gauge field and study the self-dual solitons. We also discuss 
large spin limit. In Section 3, we quantize the gauged non-compact spin system and derive the anomalous commutation relations. Section 4 is the conclusion and discussion.

\section{Noncompact Spin and CS Solitons}

We first recall that the action principle for the $(2+1)$-dimensional generalized Heisenberg ferromagnet model defined on the coadjoint orbit $G / H$ is given by [1]

$$
S=\int d t d^{2} x \operatorname{tr}\left[2 K g^{-1} \dot{g}-\partial_{i}\left(g K g^{-1}\right) \partial_{i}\left(g K g^{-1}\right)\right]
$$

where $g$ is a map $g: R^{2+1} \rightarrow G$ for the group $S U(1,1)$, and $K=i \frac{J}{2} \operatorname{diag}(1,-1) . J$ is related with the representation of $S U(1,1)$ group [19]. Let us express the element $g$ of $S U(1,1)$ by the kets $\left(\left|\Psi_{1}\right\rangle,\left|\Psi_{2}\right\rangle\right)$ with $\left|\Psi_{p}\right\rangle=\left(\Psi_{p}^{(1)}, \Psi_{p}^{(2)}\right)^{T}(p=1,2)$, and denote the inner product between the ket and its bra $\left\langle\bar{\Psi}_{q}\right|=\left(\Psi_{q(1)}^{*}, \Psi_{q(2)}^{*}\right)$ via $\left\langle\bar{\Psi}_{q} \mid \Psi_{p}\right\rangle \equiv$ $\sum_{i=1,2} \Psi_{q(i)}^{*} \Psi_{p}^{(i)}$. We introduce the metric $M^{i j}=M_{i j}=\operatorname{diag}(1,-1) \quad(i, j=1,2)$ with respect to which the raising and lowering of the indices of the components of the bra and ket are performed: $\Psi_{p}^{*(i)}=M^{i j} \Psi_{p(j)}^{*}$ and $\Psi_{p(i)}=M_{i j} \Psi_{p}^{(j)}$. Then, the inner product can be written as $\left\langle\bar{\Psi}_{q} \mid \Psi_{p}\right\rangle=\sum_{i=1,2} \Psi_{q}^{*(i)} M_{i j} \Psi_{p}^{(j)}$. The $S U(1,1)$ condition, $g^{\dagger} M g=M$ gives

$$
\left\langle\bar{\Psi}_{p} \mid \Psi_{q}\right\rangle=M_{p q}, \operatorname{det}\left(\left|\Psi_{1}\right\rangle,\left|\Psi_{2}\right\rangle\right)=1 .
$$

By making use of the second equation of (2), $\left|\Psi_{2}\right\rangle$ can be eliminated and the non-compact spin variable $Q$ can be expressed by

$$
Q \equiv g K g^{-1}=i J\left(\left|\Psi_{1}\right\rangle\left\langle\bar{\Psi}_{1}\right|-\frac{1}{2} I\right)=Q^{a} t^{b} \eta_{a b}
$$

where $t^{a}$ 's are given by the Pauli matrices; $t^{1}=-\frac{1}{2} \sigma_{2}, t^{2}=-\frac{1}{2} \sigma_{1}, t^{3}=i \frac{1}{2} \sigma_{3}$. They satisfy the Lie algebra: $\left[t^{1}, t^{2}\right]=-t^{3},\left[t^{2}, t^{3}\right]=t^{1},\left[t^{3}, t^{1}\right]=t^{2}$ and $\operatorname{tr}\left(t^{a} t^{b}\right)=$ $-\frac{1}{2} \eta^{a b}, \eta^{a b}=\operatorname{diag}(-1,-1,1)$, with the constraint $\left\langle\bar{\Psi}_{1} \mid \Psi_{1}\right\rangle=1$. This constraint can be solved explicitly in terms of the complex projective coordinate defined by $\psi^{*}=\Psi_{1}^{(2)} / \Psi_{1}^{(1)}\left(\Psi_{1}^{(1)} \neq 0\right)$ with a real gauge condition;

$$
\chi=\frac{1}{2}\left(\Psi_{1}^{*(1)}-\Psi_{1}^{(1)}\right)=0 .
$$

Then, the solution to the above constraint is given by

$$
\Psi_{1}^{*(1)}=\Psi_{1}^{(1)}=\frac{1}{\sqrt{1-|\psi|^{2}}} .
$$


Putting everything together into (11), we obtain the following reduced Lagrangian:

$$
\mathcal{L}_{\psi}=i J \frac{\psi^{*} \dot{\psi}-\dot{\psi}^{*} \psi}{1-|\psi|^{2}}-J^{2} \frac{2 \partial_{i} \psi^{*} \partial_{i} \psi}{\left(1-|\psi|^{2}\right)^{2}},
$$

and the non-compact spin from (3) follows that

$$
Q^{1}=J \frac{\psi+\psi^{*}}{1-|\psi|^{2}}, \quad Q^{2}=i J \frac{\psi^{*}-\psi}{1-|\psi|^{2}}, \quad Q^{3}=J \frac{1+|\psi|^{2}}{1-|\psi|^{2}} .
$$

We have $Q^{a} Q^{b} \eta_{a b}=J^{2}$, and $J$ is the total spin of the system.

Let us study the self-dual solitons of the above system. The Hamiltonian can be written as the Bogomol'nyi form as follows:

$$
\begin{aligned}
H & =\int d^{2} x \frac{2 J^{2} \partial_{i} \psi^{*} \partial_{i} \psi}{\left(1-|\psi|^{2}\right)^{2}}=\int d^{2} x \frac{2 J^{2}\left|\left(\partial_{1} \pm i \partial_{2}\right) \psi\right|^{2}}{\left(1-|\psi|^{2}\right)^{2}} \mp 4 \pi T, \\
T & =\frac{1}{4 \pi} \int d^{2} x\left[i J^{2} \epsilon_{i j} \partial_{i}\left(\frac{\psi^{*} \partial_{j} \psi-\psi \partial_{j} \psi^{*}}{1-|\psi|^{2}}\right)\right] .
\end{aligned}
$$

We find that the energy is saturated, when $\psi$ is self-dual or anti-self dual, but these are singular solutions because of $|\psi|=1$. Both the energy and topological charge diverge. We could restrict to the upper sheeted hyperboloid given by $|\psi|<1$ or the lower sheeted hyperboloid by $|\psi|>1$ from the beginning, but nothing prevents them from developing a divergence coming from the boundary $|\psi|=1$ in the integration. We find that the CS gauge field enables to circumvent this situation by providing an effective potential in which the boundary $|\psi|=1$ can not be reached and make the energy and topological charge finite (see (22) and Fig. 1 in which the boundary $|\psi|=1$ corresponds to $\phi=0)$.

To see this, let us consider the gauged action of (6)

$$
\begin{aligned}
S= & \int d t d^{2} x\left[i J \frac{\psi^{\dagger}\left(D_{0} \psi\right)-\left(D_{0} \psi\right)^{\dagger} \psi}{1-|\psi|^{2}}-\frac{2 J^{2}\left(D_{i} \psi\right)^{\dagger} D_{i} \psi}{\left(1-|\psi|^{2}\right)^{2}}-\gamma_{0} A_{0}-V(\psi)\right. \\
& \left.+\frac{\kappa}{2} \epsilon^{\mu \nu \rho} A_{\mu} \partial_{\nu} A_{\rho}\right]
\end{aligned}
$$

where $D_{\mu}=\partial_{\mu}-i A_{\mu}$, and $A_{\mu}$ is the CS gauge field, and $\gamma_{0}$ is the constant background charge density. We choose the potential energy to be

$$
V(\psi)=\frac{\lambda}{2}\left(-Q^{3}+\gamma\right)\left(Q^{3}-v\right),
$$

with $\gamma=\gamma_{0}+J$ and $Q^{3}$ being given as in (7). $\lambda$ is an anisotropic constant, and $v$ is a free parameter connected with symmetry breaking [20]. This specific choice of 
(10) is for the purpose of the self-duality. The Euler-Lagrange equation in terms of the non-compact spin variables $Q^{a}$ becomes

$$
D_{t} Q-D_{i}\left[Q, D_{i} Q\right]+\lambda\left(Q^{3}-\frac{v+\gamma}{2}\right)\left[Q, t^{3}\right]=0
$$

The gauge field equation is given by

$$
\frac{\kappa}{2} \epsilon^{\mu \nu \rho} F_{\nu \rho}=j^{\mu}
$$

Here $j^{\mu}$ is a conserved current expressed by

$$
j^{\mu} \equiv\left(\rho, j^{i}\right)=\left(-Q^{3}+\gamma, 2 \operatorname{tr}\left(t^{3}\left[Q, D_{i} Q\right]\right)\right)
$$

and then $Q_{\mathrm{U}(1)}=\int d^{2} x Q^{3}$ is the conserved $U(1)$ charge.

Let us first construct the Bogomol'nyi bound. Using the identity

$$
\begin{aligned}
\frac{\left(D_{i} \psi\right)^{\dagger} D_{i} \psi}{\left(1-|\psi|^{2}\right)^{2}}= & \frac{\left|\left(D_{1} \pm i D_{2}\right) \psi\right|^{2}}{\left(1-|\psi|^{2}\right)^{2}} \mp \frac{1}{2} \epsilon_{i j} F_{i j}\left(\frac{1}{1-|\psi|^{2}}-v^{\prime}\right) \\
& \mp \frac{i}{2} \epsilon_{i j} \partial_{i}\left(\frac{\psi^{*} \partial_{j} \psi-\psi \partial_{j} \psi^{*}}{1-|\psi|^{2}}\right) \pm \epsilon_{i j} \partial_{i}\left(\left(\frac{1}{1-|\psi|^{2}}-v^{\prime}\right) A_{j}\right)
\end{aligned}
$$

where $v^{\prime}$ is an arbitrary constant, we obtain the following Bogomol'nyi equation:

$$
\begin{aligned}
H & =\int d^{2} x\left(\frac{2 J^{2}\left|D_{i} \psi\right|^{2}}{\left(1-|\psi|^{2}\right)^{2}}+V(\psi)\right) \\
& =\int d^{2} x\left[\frac{2 J^{2}\left|\left(D_{1} \pm i D_{2}\right) \psi\right|^{2}}{\left(1-|\psi|^{2}\right)^{2}}+V(\psi) \mp \frac{J}{2} \epsilon_{i j} F_{i j}\left(Q^{3}-v^{\prime \prime}\right)\right] \mp 4 \pi T,
\end{aligned}
$$

where the topological charge is given by

$$
4 \pi T=\int d^{2} x\left[i J^{2} \epsilon_{i j} \partial_{i}\left(\frac{\psi^{*} \partial_{j} \psi-\psi \partial_{j} \psi^{*}}{1-|\psi|^{2}}\right)-J \epsilon_{i j} \partial_{i}\left(\left(Q^{3}-v^{\prime \prime}\right) A_{j}\right)\right],
$$

with $v^{\prime \prime}=\left(2 v^{\prime}-1\right) J$.

Using the Gauss Law constraint

$$
\frac{\kappa}{2} \epsilon_{i j} F_{i j}=-Q^{3}+\gamma
$$

and from (10) and (15), we find that self-dual limit is achieved with the condition $\lambda= \pm \frac{2 J}{\kappa}$ and $v=v^{\prime \prime}\left(=\left(2 v^{\prime}-1\right) J\right)$. Note that the latter condition connects the symmetry breaking parameter with the topological charge. The Bogomol'nyi 
bound is saturated by the topological charge when the self-duality equation is satisfied:

$$
\left(D_{1} \pm i D_{2}\right) \psi=0 \text {. }
$$

Expressing the gauge field $A_{i}$ in terms of $\psi=|\psi| e^{i \Theta}$, we find

$$
A_{j}=\partial_{j} \Theta \pm \epsilon_{j k} \partial_{k} \ln |\psi|
$$

Substitution into Gauss's law (17) yields

$$
\nabla^{2} \ln |\psi| \pm \epsilon^{i j} \partial_{i} \partial_{j} \Theta= \pm \frac{1}{\kappa}\left(J \frac{1+|\psi|^{2}}{1-|\psi|^{2}}-\gamma\right)
$$

We concentrate on $J=1$ case for the time being. It will be restored later when we discuss large $J$ limit. Let us define $|\psi| \equiv e^{-\phi}$, then we obtain a scalar equation for the soliton configuration:

$$
\nabla^{2} \phi \pm \epsilon^{i j} \partial_{i} \partial_{j} \Theta=-\frac{d V_{e f f}}{d \phi}
$$

where the "effective" potential $V_{\text {eff }}$ is given by (See Fig. 1)

$$
V_{e f f}(\phi)= \pm \frac{1}{\kappa}(\ln \sinh \phi-\gamma \phi) \text {. }
$$

Let us concentrate on the upper sign (self-dual case) and the rotationally symmetric solutions. The lower sign can be handled in a similar fashion. The ansatz in the cylindrical coordinate $(r, \theta)$ is given by

$$
\phi=\phi(r), \Theta=n \theta, A_{i}=-\frac{\epsilon_{i j} x_{j}}{r^{2}} a(r)
$$

Then, the Gauss's law (17) and self-dual equation (18) are given as follows:

$$
\begin{aligned}
r f^{\prime}(r) & =[n-a(r)] \sinh f(r), \\
a^{\prime}(r) & =-\frac{r}{\kappa}[\cosh f(r)-\gamma] .
\end{aligned}
$$

Also the equation of motion in (21) becomes an analogue of the one dimensional Newton's equation for $r>0$, if we regard $r$ as "time" and $\phi$ as the position of the hypothetical particle with unit mass:

$$
\frac{d^{2} \phi}{d r^{2}}+\frac{1}{r} \frac{d \phi}{d r}=\frac{1}{\kappa}(-\operatorname{coth} \phi+\gamma) .
$$


The exerting forces are the conservative force from the effective potential $V_{\text {eff }}(\phi)$ in (22), and time-dependent friction $\frac{1}{r} \frac{d \phi}{d r}$. When $n \neq 0$, there is an impact term at $r=0$ due to $\epsilon^{i j} \partial_{i} \partial_{j} n \theta=\frac{n}{r} \delta(r)$ in (21).

The effective potential suggests that solutions are of three types; the topological vortex with $n \neq 0(\kappa>0, \gamma>1)$, the non-topological vortex with $n \neq 0(\kappa<$ $0, \gamma=1)$, and the non-topological soliton with $n=0(\kappa<0, \gamma=1)$. In the first case, the particle starts from $\phi=\infty$ at $r=0$ and moves until it reaches $\phi=\operatorname{coth}^{-1} \gamma$ at $r=\infty$. It corresponds to broken vacuum with $Q^{3}=\gamma$. In the second case, the particle starts from $\phi=\infty$, reaches a turning point where it stops, changes the direction, and finally rolls down again to $\phi=\infty$. In the last case, the particle starting at some finite position, rolls down directly to $\phi=\infty$. Let us examine the behavior of the solutions more closely. Near $r=0$, the condition for $A_{i}$ to be non-singular forces $a(0)=0$ and $n f(0)=0$. Hence when $n \neq 0, f(0)=0$ and when $n=0, f(0)$ is arbitrary. The behaviors of the solutions near $r=\infty$ are determined by the condition $f^{\prime}(\infty)=a^{\prime}(\infty)=0$.

I. Topological vortex $(\kappa>0, \gamma>1)$.

Near $r=0$, trying power solutions of the form $f(r)=f_{0} r^{p}, a(r)=a_{0} r^{q}$, we find $p=n$ (positive), $q=2$, and $a_{0}=\frac{\gamma-1}{2 \kappa}$. (24) forces $f^{\prime}(r)>0$ near $r=0$. Hence $a(r)$ and $f(r)$ are monotonically increasing function near $r=0$. Near $r=\infty$, since $\phi(\infty)=\operatorname{coth}^{-1} \gamma, f(\infty)$ is $\ln \left(\gamma+\sqrt{\gamma^{2}-1}\right)$ and the finiteness of energy requires $a(\infty)=n$.

II. Non-topological vortex $(\kappa<0, \gamma=1, n>0)$.

Near $r=0$, we find $f=f_{0} r^{n}$ and $a(r)=-\frac{f_{0}^{2}}{4(n+1) \kappa} r^{2 n+2}$ which are all monotonically increasing functions near $r=0$. Near $r=\infty$, let us try $f(r)=f_{\infty} r^{-p}, a(r)=$ $\alpha+a_{\infty} r^{-q}$ where $\alpha \equiv a(\infty)$. Then we find $p=-n+\alpha, q=2(\alpha-n-1)$, hence $\alpha>n+1$.

III. Non-topological soliton $(\kappa<0, \gamma=1, n=0)$.

Near $r=0$, let us try $f(r)=f(0)+f_{0} r^{p}$ and $a(r)=a_{0} r^{q}$. We find $p=q=2$ and $f_{0}>0$. This means that no solution exists in which the "particle" climbs up the hill at first. It rolls down from the beginning. Hence $f(r)$ is a monotonically decreasing function and $a(r)$ is an increasing function. Near $r=\infty$, let us try $f(r)=f_{\infty} r^{-p}, a(r)=\alpha+a_{\infty} r^{-q}$. We find $p=\alpha, q=2(\alpha-1)$ and $a_{\infty}<0$, hence $\alpha>1$.

Some numerical results are presented in Fig. 2 and 3. 
Let us compute physical quantities of these solitons under the ansatz in (23). The topological charge (16) can be expressed by

$$
T=\frac{n}{2}(\cosh f(0)-\cosh f(\infty))-\frac{\alpha}{2}\left(\cosh f(\infty)-v^{\prime \prime}\right) .
$$

For topological vortex, we find $T=\frac{n}{2}\left(1-2 \gamma+v^{\prime \prime}\right)$ and for nontopological vortex and soliton, $T=-\frac{\alpha}{2}\left(1-v^{\prime \prime}\right)$. The magnetic flux $\Phi \equiv \int d^{2} x B$ is given by

$$
\Phi=2 \pi \alpha
$$

It is $2 \pi n$ for the topological vortex, and $2 \pi(n+\beta)$, with $\beta=\alpha-n$, for others. We define the angular momentum 21]

$$
M=\int d^{2} x \epsilon_{i j} x_{i}\left\{i \frac{\left(\partial_{j} \psi^{*}\right) \psi-\psi^{*}\left(\partial_{j} \psi\right)}{1-|\psi|^{2}}-A_{j}\left(Q^{3}-1\right)\right\} .
$$

We count only the unambiguous contribution coming from the gauge field in the absence of the background charge $\gamma_{0}$. It is given by

$$
M=\pi \kappa \alpha(\alpha-2 n)
$$

$M$ is in general fractional, which means the solitons are anyons. It tells that $M=-\pi \kappa n^{2}$ for topological vortex, $M=\pi \kappa\left(\beta^{2}-n^{2}\right)$ for nontopological vortex, and $M=\pi \kappa \beta^{2}$ for nontopological soliton.

Next, we examine the large $J$ limit. To do that, let us consider the HolsteinPrimakoff transformation and introduce $u$ defined by

$$
u=\frac{\sqrt{2 J}|\psi|}{\sqrt{1-|\psi|^{2}}}
$$

Substitution of the inverse transformation

$$
|\psi|=\frac{u}{\sqrt{2 J+u^{2}}}
$$

into (20) yields

$$
\nabla^{2} \ln u-\frac{1}{2} \nabla^{2} \ln \left(2 J+u^{2}\right)= \pm \frac{1}{\kappa}\left(u^{2}-\gamma+J\right) .
$$

We find that in the limit $J \rightarrow \infty$ and $\gamma=J$, neglecting terms of order $1 / J$, the equation is precisely the Liouville equation of the gauged nonlinear Schrödinger model [16]. Also when $-\gamma+J=1$ it reduces to the Abelian Higgs model [17. 


\section{Quantization}

In this section, we consider quantization of the gauged spin system. Since the Lagrangian of our model is singular, we use the Dirac's constraint analysis [22], together with the first-order method advocated by Faddeev and Jackiw [23. We first represent the action (9) in terms of the canonical variables $(f, \Theta)$ given by $\psi=\tanh \frac{f}{2} e^{i \Theta}$. To do that, we use the non-compact spin variables;

$$
Q^{1}=J \sinh f(\mathbf{r}) \cos \Theta(\mathbf{r}), Q^{2}=J \sinh f(\mathbf{r}) \sin \Theta(\mathbf{r}), Q^{3}=J \cosh f(\mathbf{r}) .
$$

We denote vectors with bold face from here on. The first order Lagrangian can be rewritten as [23]

$$
L=a_{I}(\xi) \dot{\xi}^{I}-H(\xi)
$$

where $\xi^{I}$ denotes collectively the canonical variables, $\left(f(\mathbf{r}), \Theta(\mathbf{r}), A_{i}(\mathbf{r})\right)$. The time dependence will be omitted unless needed. The Hamiltonian (15) with the Gauss's law constraint is given by

$$
H=\int d^{2} \mathbf{r}\left[\frac{J^{2}}{2}\left(\left(\partial_{i} f(\mathbf{r})\right)^{2}+\sinh ^{2} f(\mathbf{r})\left(\partial_{i} \Theta-A_{i}\right)^{2}\right)+V+A^{0} \tilde{G}\right] .
$$

The potential $V$ is given in (10) and the Gauss's law is implemented as the first class constraint;

$$
\tilde{G}=\frac{\kappa}{2} \epsilon_{i j} F_{i j}+Q_{3}-\gamma \approx 0 .
$$

The Euler-Lagrangian equation from (35) reads as

$$
\Omega_{I J}(\xi) \dot{\xi}^{J}=\frac{\partial H(\xi)}{\partial \xi^{I}}
$$

with $\Omega_{I J}(\xi)=\frac{\partial}{\partial \xi^{I}} a_{J}(\xi)-\frac{\partial}{\partial \xi^{J}} a_{I}(\xi) . \Omega_{I J}(\xi)$ defines the pre-symplectic two form $\Omega$ by

$$
\Omega=\frac{1}{2} \Omega_{I J}(\xi) d \xi^{I} d \xi^{J}=d a(\xi),
$$

where $a(\xi)$ is the canonical one form, $a(\xi)=a_{I}(\xi) d \xi^{I}$. For the given first order Lagrangian, we find

$$
\Omega=\int d^{2} \mathbf{r}\left(J \sinh f(\mathbf{r}) d f(\mathbf{r}) d \Theta(\mathbf{r})+\frac{\kappa}{2} \epsilon^{i j} d A_{i}(\mathbf{r}) d A_{j}(\mathbf{r})\right) .
$$


When the matrix $\left(\Omega_{I J}\right)$ is non-singular, i.e., has its inverse $\left(\Omega^{I J}\right)$ as in $(40)$, the Poisson bracket is defined as

$$
\begin{aligned}
& \{F(\xi), G(\xi)\}=\Omega^{I J}(\xi) \frac{\partial F(\xi)}{\partial \xi^{I}} \frac{\partial G(\xi)}{\partial \xi^{J}} \\
& =\int d^{2} \mathbf{r}\left[\frac{1}{J \sinh f(\mathbf{r})}\left(\frac{\partial F}{\partial f(\mathbf{r})} \frac{\partial G}{\partial \Theta(\mathbf{r})}-\frac{\partial F}{\partial \Theta(\mathbf{r})} \frac{\partial G}{\partial f(\mathbf{r})}\right)+\left(\frac{1}{\kappa} \epsilon_{i j} \frac{\partial F}{\partial A_{i}(\mathbf{r})} \frac{\partial G}{\partial A_{j}(\mathbf{r})}\right)\right],
\end{aligned}
$$

and the Euler-Lagrangian equations become

$$
\dot{\xi}^{I}=\left\{\xi^{I}, H\right\}=\Omega^{I J} \frac{\partial H}{\partial \xi^{J}} .
$$

The above Poisson bracket yields

$$
\begin{aligned}
\left\{J \cosh f(\mathbf{r}), \Theta\left(\mathbf{r}^{\prime}\right)\right\} & =\delta\left(\mathbf{r}-\mathbf{r}^{\prime}\right) \\
\left\{A_{i}(\mathbf{r}), A_{j}\left(\mathbf{r}^{\prime}\right)\right\} & =\frac{1}{\kappa} \epsilon_{i j} \delta\left(\mathbf{r}-\mathbf{r}^{\prime}\right) .
\end{aligned}
$$

Then, by using the expression (34), we recover the $s u(1,1)$ spin algebra

$$
\left\{Q^{a}(\mathbf{r}), Q^{b}\left(\mathbf{r}^{\prime}\right)\right\}=-\epsilon^{a b}{ }_{c} Q^{c}(\mathbf{r}) \delta\left(\mathbf{r}-\mathbf{r}^{\prime}\right) .
$$

One can check that no further secondary constraints arise

$$
\{H, \tilde{G}\} \approx 0
$$

and (42) yields the equations of motions (11) and (12).

To quantize the system, we adopt the scheme which has two properties. The first one is that we pursue the quantization of dynamical variables which are functions of only $Q^{a}$ 's, which satisfy the basic $s u(1,1)$ algebra (45). This is due to the fact that not every observables which are functions of the canonical variables satisfying (43) are quantizable 24]. Therefore, we prepare the classical Hamiltonian in the form;

$$
H=\int d^{2} \mathbf{r}\left[\frac{1}{2}\left(D_{i} Q^{1} \cdot D_{i} Q^{1}+D_{i} Q^{2} \cdot D_{i} Q^{2}-D_{i} Q^{3} \cdot D_{i} Q^{3}\right)+V\left(Q^{3}\right)+A^{0} \tilde{G}\right]
$$

Then, we introduce $Q_{ \pm}=Q^{1} \pm i Q^{2}, Q_{0}=Q^{3}$ and use the canonical relations coming from quantizing the classical algebra (45);

$$
\left[Q_{+}(\mathbf{r}), Q_{-}\left(\mathbf{r}^{\prime}\right)\right]=-2 Q_{0}(\mathbf{r}) \delta\left(\mathbf{r}-\mathbf{r}^{\prime}\right),\left[Q_{0}(\mathbf{r}), Q_{ \pm}\left(\mathbf{r}^{\prime}\right)\right]= \pm Q_{ \pm}(\mathbf{r}) \delta\left(\mathbf{r}-\mathbf{r}^{\prime}\right)
$$


Secondly, we work in the reduced phase space quantization scheme [16] in which the Gauss's law is first solved to yield the quantum gauge potential $\left(\nabla=\frac{\partial}{\partial \mathbf{r}}\right)$

$$
\mathbf{A}(\mathbf{r})=\nabla \times \frac{1}{\kappa} \int d^{2} \mathbf{r}^{\prime} G\left(\mathbf{r}-\mathbf{r}^{\prime}\right)\left[-Q_{0}\left(\mathbf{r}^{\prime}\right)+\gamma\right]
$$

where $G\left(\mathbf{r}-\mathbf{r}^{\prime}\right)$ is the Green's function;

$$
G\left(\mathbf{r}-\mathbf{r}^{\prime}\right)=\frac{1}{2 \pi} \ln \left|\mathbf{r}-\mathbf{r}^{\prime}\right|
$$

We will take $\mathbf{A}(\mathbf{r})$ to commute with $Q_{ \pm}(\mathbf{r}), Q_{0}(\mathbf{r})$ [16].

Then, the normal ordered Hamiltonian is given by

$$
H=\int d^{2} \mathbf{r}\left(\frac{1}{2} \boldsymbol{\Pi}^{\dagger} \cdot \boldsymbol{\Pi}-\frac{1}{2} \nabla Q_{0} \cdot \nabla Q_{0}+\frac{\lambda}{2}\left(-Q_{0}+\gamma\right)\left(Q_{0}-v\right)+A^{0} \tilde{G}\right)
$$

where

$$
\Pi=\mathbf{D} Q_{-} \equiv \nabla Q_{-}-i \mathbf{A} Q_{-} .
$$

The equation of motion is given by

$$
\begin{aligned}
i \dot{Q}_{-}(\mathbf{r})= & {\left[Q_{-}(\mathbf{r}), H\right] } \\
= & -Q_{0}(\mathbf{r}) D^{2} Q_{-}(\mathbf{r})+\frac{1}{2}\left[Q_{-}(\mathbf{r}), \nabla^{2} Q_{0}(\mathbf{r})\right]_{+}-A^{0}(\mathbf{r}) Q_{-}(\mathbf{r}) \\
& +\frac{1}{2 \kappa^{2}} \int d^{2} \mathbf{r}^{\prime} \nabla G\left(\mathbf{r}-\mathbf{r}^{\prime}\right) \cdot \nabla G\left(\mathbf{r}-\mathbf{r}^{\prime}\right) Q_{+}\left(\mathbf{r}^{\prime}\right) Q_{-}\left(\mathbf{r}^{\prime}\right) Q_{-}(\mathbf{r}) \\
& -\frac{\lambda}{2}\left(\left[Q_{-}(\mathbf{r}), Q_{0}(\mathbf{r})\right]_{+}-(\gamma+v) Q_{-}(\mathbf{r})\right),
\end{aligned}
$$

where $[,]_{+}$denotes anticommutator. The scalar potential $A^{0}$ is given by

$$
A^{0}(\mathbf{r})=-\frac{1}{\kappa} \int d^{2} \mathbf{r}^{\prime} \quad G\left(\mathbf{r}-\mathbf{r}^{\prime}\right) \nabla \times \mathbf{j}\left(\mathbf{r}^{\prime}\right)
$$

where $\mathbf{j}$ is the current-density operator

$$
\mathbf{j}=\frac{1}{2 i}\left[Q_{+}(\mathbf{r}) \boldsymbol{\Pi}(\mathbf{r})-\boldsymbol{\Pi}^{\dagger}(\mathbf{r}) Q_{-}(\mathbf{r})\right]
$$

The third component $Q_{0}$ satisfies the current conservation equation:

$$
\dot{Q}_{0}+\nabla \cdot \mathbf{j}=0
$$

To give anyon interpretation, let us define a new operator

$$
\hat{Q}_{-}(\mathbf{r})=e^{-i \omega(\mathbf{r})} Q_{-}(\mathbf{r}) \equiv \exp \left[\frac{i}{2 \pi \kappa} \int d^{2} \mathbf{r}^{\prime} \Sigma\left(\mathbf{r}-\mathbf{r}^{\prime}\right)\left(-Q_{0}\left(\mathbf{r}^{\prime}\right)+\gamma\right)\right] Q_{-}(\mathbf{r})
$$


where $\Sigma$ is given by

$$
\nabla \times G\left(\mathbf{r}-\mathbf{r}^{\prime}\right)=-\frac{1}{2 \pi} \nabla \Sigma\left(\mathbf{r}-\mathbf{r}^{\prime}\right), \quad \tan \Sigma\left(\mathbf{r}-\mathbf{r}^{\prime}\right)=\frac{y-y^{\prime}}{x-x^{\prime}} .
$$

We also define $\hat{Q}_{0}(\mathbf{r})=Q_{0}(\mathbf{r})$. A straightforward computation shows that the new operators satisfy the anomalous commutator

$$
\begin{aligned}
\hat{Q}_{+}(\mathbf{r}) \hat{Q}\left(\mathbf{r}^{\prime}\right) & =e^{\frac{i}{2 \kappa}}\left[\hat{Q}_{-}\left(\mathbf{r}^{\prime}\right) \hat{Q}_{+}(\mathbf{r})-2 \hat{Q}_{0}(\mathbf{r}) \delta\left(\mathbf{r}-\mathbf{r}^{\prime}\right)\right] \\
\hat{Q}_{ \pm}(\mathbf{r}) \hat{Q}_{ \pm}\left(\mathbf{r}^{\prime}\right) & =e^{-\frac{i}{2 \kappa}} \hat{Q}_{ \pm}\left(\mathbf{r}^{\prime}\right) \hat{Q}_{ \pm}(\mathbf{r}) \\
{\left[\hat{Q}_{0}(\mathbf{r}), \hat{Q}_{ \pm}\left(\mathbf{r}^{\prime}\right)\right] } & = \pm \hat{Q}_{ \pm}(\mathbf{r}) \delta\left(\mathbf{r}-\mathbf{r}^{\prime}\right)
\end{aligned}
$$

Using the identity 16

$$
-i\left[\omega(\mathbf{r}), \partial_{t} \omega(\mathbf{r})\right]=\frac{1}{4 \pi^{2} \kappa^{2}} \int d^{2} \mathbf{r}^{\prime} \nabla \Sigma\left(\mathbf{r}-\mathbf{r}^{\prime}\right) \cdot \nabla \Sigma\left(\mathbf{r}-\mathbf{r}^{\prime}\right) Q_{+}\left(\mathbf{r}^{\prime}\right) Q_{-}\left(\mathbf{r}^{\prime}\right),
$$

one can show that (53) becomes "free" equation in terms of $\hat{Q}$;

$$
\begin{aligned}
i \dot{\hat{Q}}_{-}(\mathbf{r})= & -\hat{Q}_{0}(\mathbf{r}) \nabla^{2} \hat{Q}_{-}(\mathbf{r})+\frac{1}{2}\left[\hat{Q}_{-}(\mathbf{r}), \nabla^{2} \hat{Q}_{0}(\mathbf{r})\right]_{+} \\
& -\frac{\lambda}{2}\left(\left[\hat{Q}_{-}(\mathbf{r}), \hat{Q}_{0}(\mathbf{r})\right]_{+}-(\gamma+v) \hat{Q}_{-}(\mathbf{r})\right) .
\end{aligned}
$$

Hence, the our system provides another field theoretical model of anyons [25]: the gauge potential can be eliminated by a singular gauge transformation, and the multivalued operators $\hat{Q}$ 's satisfy a free equation but anomalous commutation relations.

To consider the large spin limits, let us introduce the Holstein-Primakoff transformation:

$$
\begin{aligned}
Q_{-}(\mathbf{r}) & =\sqrt{2 J+\psi^{\dagger}(\mathbf{r}) \psi(\mathbf{r})} \psi(\mathbf{r}), \quad Q_{+}(\mathbf{r})=\psi^{\dagger}(\mathbf{r}) \sqrt{2 J+\psi^{\dagger}(\mathbf{r}) \psi(\mathbf{r})}, \\
Q_{0}(\mathbf{r}) & =J+\psi^{\dagger}(\mathbf{r}) \psi(\mathbf{r})
\end{aligned}
$$

One can check that the algebra (48) is satisfied if one postulates the following commutator [26]:

$$
\left[\psi(\mathbf{r}), \psi^{\dagger}\left(\mathbf{r}^{\prime}\right)\right]=\delta\left(\mathbf{r}-\mathbf{r}^{\prime}\right),\left[\psi(\mathbf{r}), \psi\left(\mathbf{r}^{\prime}\right)\right]=\left[\psi^{\dagger}(\mathbf{r}), \psi^{\dagger}\left(\mathbf{r}^{\prime}\right)\right]=0
$$

In the large $J$ limit, let us rescale $\lambda \rightarrow 2 J \lambda, A^{0} \rightarrow 2 J A^{0}$, and choose $\gamma=v=J$. Then, substituting (62) into (51) and keeping only the linear terms in $J$, we obtain (after we rescale $H$ by $2 J H$, and perform normal ordering with respect to $\psi$ )

$$
H=\int d^{2} \mathbf{r}\left[\frac{1}{2} \boldsymbol{\Pi}^{\dagger} \cdot \boldsymbol{\Pi}-\frac{\lambda}{2}\left(\psi^{\dagger} \psi\right)^{2}+A^{0}\left(\frac{\kappa}{2} \epsilon_{i j} F_{i j}+|\psi|^{2}\right)\right],
$$


which is the Hamiltonian of the gauged non-linear Schrödinger model of Jackiw and $\mathrm{Pi}[16]$.

\section{Conclusion}

We found that the gauged non-compact spin system admits various self-dual rotationally symmetric solitons. Depending on the background charge, they can be classified as the topological vortices, the nontopological vortices, and the nontopological solitons. The topological lump solutions do not exist because of the non-compact nature of the target space. These soliton equations have hidden large spin limits which can be reached by the classical Holstein-Primakoff realization. By a suitable choice of the background charges, the system reduces to the Liouville equation whose exact multi-soliton solutions are known, or the Abelian Higgs model. In the second quantum aspect, we found that the quantization of the gauged non-compact spin system leads to the nonrelativistic field theory describing anyons, and its large spin limit is also connected with the gauged nonlinear Schrödinger model. This provides yet another realization of the CS field producing the long-range interaction which is responsible for the fractional statistics.

There remain several issues which deserve further investigation. The first one is related with avoiding the singularity $|\psi|=1$ to obtain finite energy solitons. We found that CS gauge field makes this possible by providing an effective potential $V_{\text {eff }}$ of (22). This corresponds to introducing a scale into the flat potential. In this respect, there remains other alternatives, especially inclusion of the fourth-order spatial derivative Skyrmion term [27]. This term does not permit any self-duality in $2+1$ dimension, but the existence of the finite energy solitons itself would be interesting (the same statement also holds in the compact case). The analytic behaviors of the self-dual solitons deserve further study. The existence of the multivortex solutions (with multicenters) [28] of the nonlinear partial differential equations described by (21) and (22) for the various background charges needs to be analyzed in detail.

The extensions into "higher" cases remain as open problems. First, we can consider higher non-compact $C P(N)$ case [29]. The pivotal thing is to check whether the self-dual equations admit solitons with finite positive semi-definite energy. Gauging the full $S U(1,1)$ group or $S U(2)$ in the compact case is another direction to try. In these cases, the Gauss's law (17) will be replaced by the non- 
Abelian version. This can be attacked either in holomorphic gauge [30 or in axial gauge [31. It would be interesting to look into the self-dual equations in these gauges to find out whether they lead to any sensible soliton theory. The quantization could also be performed in these gauges, but the non-compact case will require a more careful treatment because of the non-compact nature of the gauge field. The relations with the relativistic $S U(1,1)$ model of 11 should be studied also to see whether the present non-relativistic self-dual solitons could be obtained by taking some suitable limit.

On the more physical side, it would be meaningful if the CS gauged spin system could be realized in the parity broken system. Especially note that similar type of vortices are analyzed in the double-layer fractional quantum Hall effect, which is described by the compact $C P(1)$ nonlinear sigma model coupled with the CS gauge field and a Hopf term [32]. Since the non-compact symmetry is also connected with the quantum Hall effect [9], the vortices, solitons and anyon realization of the simplest non-compact symmetry presented in this paper could have some physical application.

\section{Acknowledgments}

We would like to thank Dr. K. Kimm for useful discussions and R. Jackiw for carefully reading the manuscript. This work is supported in part by the Korea Science and Engineering Foundation through the CTP at SNU and the project number (95-0702-04-01-3), and by the Ministry of Education through the Research Institute for Basic Science (BSRI/97-1419).

\section{References}

[1] P. Oh and Q-H. Park, Phys. Lett. B383, 333 (1996).

[2] S. Helgason, Differential Geometry, Lie Groups, and Symmetric Spaces ( Academic Press, New York, 1978).

[3] P. Oh and Q-H. Park, Phys. Lett. B400, 157 (1997); (E) 416, 452 (1998).

[4] Y. Kim, P. Oh and C. Rim, Mod. Phys. Lett. A12, 3169 (1997). 
[5] R. Jackiw, Progr. Theor. Phys. (Kyoto), Suppl. 107, 1 (1992); Recent Developments in Field Theory, J. E. Kim, ed. (Min Eum Sa, Seoul, 1992); G. Dunne, Self-Dual Chern-Simons Theories (Springer, Berlin, 1995).

[6] A. C. Davis, M. D. Freeman, and A. J. Macfarlane, Nucl. Phys. B258, 393 (1985); A. C. Davis, A. J. Macfarlane and J. W. van Holten, Phys.Lett. B125, 151 (1983); A. C. Davis, A. J. Macfarlane and J. W. van Holten, Nucl. Phys. B216, 493 (1983); ibid 232, 473 (1984); Y. Cohen and E. Rabinovichi, Phys. Lett. B124, 371 (1983); D. J. Amit and A. C. Davis, Nucl. Phys. B225 [FS9], 221 (1983).

[7] A. Kundu, Lett. Math. Phys. 6, 479 (1986); A. Kundu, J. Phys. A: Math. Gen. 19, 1303 (1986); V. G. Makhankov and O. K. Pashaev, Theor. Math. Phys. 53, 979 (1982); Y. K. Zhou, Physica Scripta 39, 680 (1989).

[8] J. Gruszcak, J. Phys. A: Math, Gen. 14, 3247 (1981); S. Takeno, Progr. Theor. Phys. 66, 1250 (1981).

[9] A. M. M. Pruisken, Nucl. B290 [FS20], 61 (1987); H. A. Weidenmüller and M. R. Zirnbauer, Nucl. Phys. B305 [FS23], 339 (1988).

[10] V. G. Makhanokov, Phys. Lett. A81, 151 (1981); V. G. Makhanokov, N. V. Makhaldiani, and O. K. Pashaev, Phys. Lett. A81, 161 (1981); V. G. Makhanokov and O. K. Pashaev, Physica Scripta 28, 229 (1983); R. Myrzakulov, O. K. Pashaev and Kh. T. Kholmurodov, Physica Scripta 33, 378 (1986).

[11] P. Oh, hep-th/9707150.

[12] G. H. Derrick, J. Math. Phys. 5, 1252 (1964); R. Hobart, Proc. Phys. Soc. Lond. 82, 201 (1963).

[13] A. A. Belavin and A. M. Polyakov, JETP Lett. 22, 245 (1975).

[14] R. A. Leo, L. Martina and G. Soliani, Phys. Lett. B247, 562 (1990); R. A. Leo, L. Martina and G. Soliani, Phys. Lett. B228, 95 (1989).

[15] P. Oh and C. Rim, Mod. Phys. Lett. A12, 163 (1997), and reference therein. 
[16] R. Jackiw and S.-P. Pi, Phys. Rev. Lett. 64, 2969 (1990); Phys. Rev. D42, 3500 (1990); (E) ibid 48, 3929 (1993).

[17] E. J. Weinberg, Phys. Rev. D19, 3008 (1979)

[18] F. Wilczek, ed., Fractional Statistics and Anyon Superconductivity (World Scientific, Singapore, 1992).

[19] A. Perelomov, Generalized Coherent States and Their Applications (SpringerVerlag, Berlin, 1986).

[20] K. Kimm, K. Lee, and T. Lee, Phys. Rev. D53, 4436 (1996).

[21] Note that the expression (29) is a gauged version of the standard angular momentum $M=\int d^{2} x \epsilon_{i j} x_{i} p_{j}$ with the momentum density $p_{j}=i \frac{\partial_{j} \psi^{*} \psi-\psi^{*} \partial_{j} \psi}{1-|\psi|^{2}}$. The total momentum $P_{j} \equiv \int d^{2} x p_{j}$ expressed in terms of $f, \theta$ is given by $P_{j}=n \int d^{2} x \sinh ^{2} \frac{f}{2} \partial_{j} \theta$. It does not suffer from the non-differentiability of the azimuthal angle unlike the compact case; N. Papanicolaou and T.N. Tomaras, Nucl. Phys. B360, 425 (1991); E.G. Floratos, Phys. Lett. B279, 117 (1992).

[22] P.A.M. Dirac, Lectures on Quantum Mechanics (Yeshiva University, New York, 1964).

[23] L. D. Faddeev and R. Jackiw, Phys. Rev. Lett. 60, 1692 (1988).

[24] This is one of the basic idea of the geometric quantization scheme; R. Abraham and J. E. Marsden, Foundations of Mechanics (Addison Wesley, 1978).

[25] F. Wilczek and A. Zee, Phys. Rev. Lett. 51, 2250 (1983); M. J. Bowick, D. Karabali, and L. C. R. Wijewardhana, Nucl. Phys. B271, 417 (1986); A. M. Polyakov, Mod. Phys. Lett. A3, 325 (1988); G. W. Semenoff, Phys. Rev. Lett. 61, 517 (1988); T. Matsuyama, Phys. Lett. B228, 99 (1989); R. Jackiw and S.-P. Pi, Ref. [16]; C.-G. Han, Phys. Rev. D47, 5521 (1993).

[26] Note that in the compact case with the spin algebra $\left[Q_{+}(\mathbf{r}), Q_{-}\left(\mathbf{r}^{\prime}\right)\right]=2 Q_{0}(\mathbf{r}) \delta\left(\mathbf{r}-\mathbf{r}^{\prime}\right),\left[Q_{0}(\mathbf{r}), Q_{ \pm}\left(\mathbf{r}^{\prime}\right)\right]= \pm Q_{ \pm}(\mathbf{r}) \delta\left(\mathbf{r}-\mathbf{r}^{\prime}\right)$, the Holstein-Primakoff realization is given by $Q_{+}(\mathbf{r})=\sqrt{2 J-\psi^{\dagger}(\mathbf{r}) \psi(\mathbf{r})} \psi(\mathbf{r})$, $Q_{-}(\mathbf{r})=\psi^{\dagger}(\mathbf{r}) \sqrt{2 J-\psi^{\dagger}(\mathbf{r}) \psi(\mathbf{r})}, Q_{0}(\mathbf{r})=J-\psi^{\dagger}(\mathbf{r}) \psi(\mathbf{r})$ and we need opposite normal ordering. 
[27] L. Faddeev and A. J. Niemi, hep-th/9705176.

[28] Y. Yang, Commun. Math. Phys. 181, 485 (1996).

[29] For example, the case of $S U(N, 1) / S U(N) \times U(1)$ can be considered which is described in [15].

[30] T. Lee and P. Oh, Ann. Phys. 235, 413 (1994).

[31] D. Bak, R. Jackiw and S.-Y. Pi, Phys. Rev. D49, 6778 (1994).

[32] I. Ichinose and A. Sekiguchi, Nucl. Phys. B493, 683 (1997); Mod. Phys. Lett. A12, 2243 (1997). 


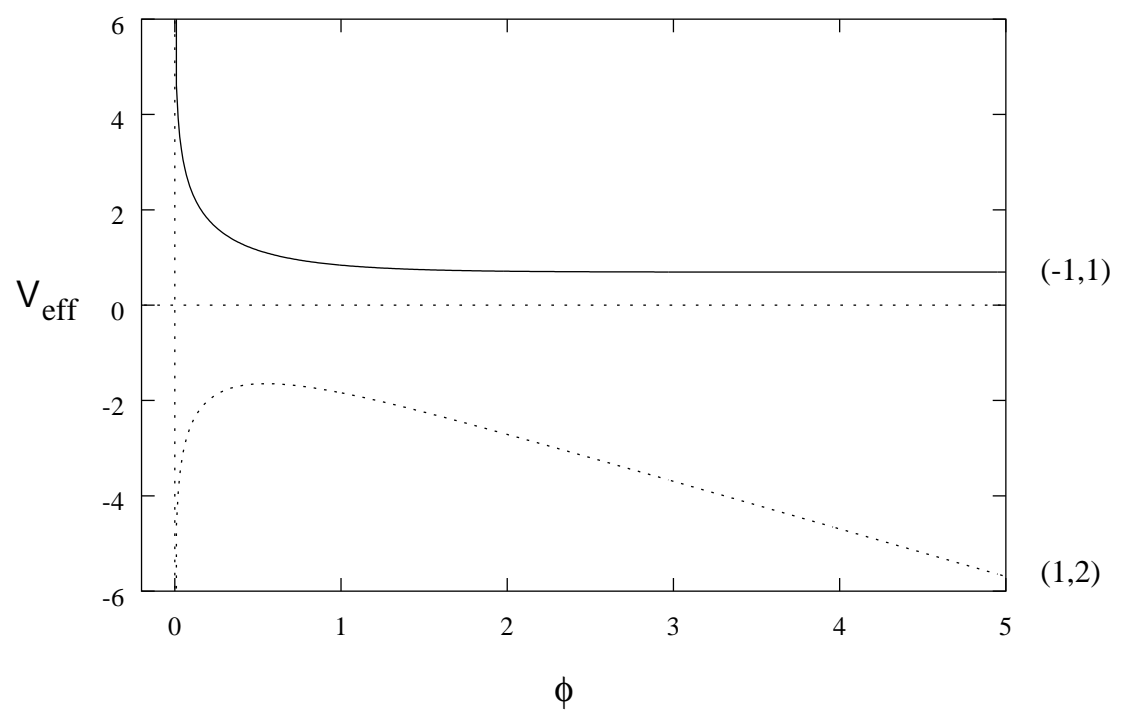

Fig.1 Shapes of $V_{\text {eff }}(\phi)$ for some values of $\kappa$ and $\gamma$, e.g., $(-1,1)$ means $\kappa=-1$ and $\gamma=1$. 


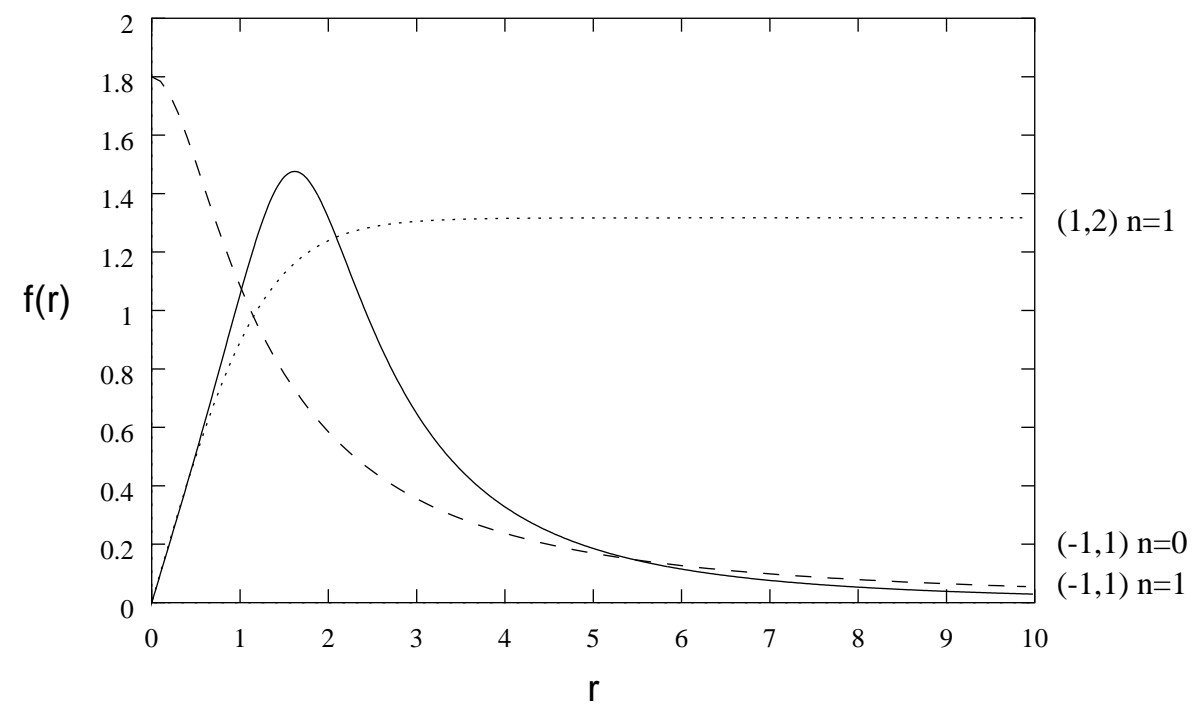

Fig.2 Shapes of $f(r)$.

The solid line denotes non-topological vortex, the dotted line topological vortex, and the dashed line non-topological soliton. $(-1,1)$ means $\kappa=-1$ and $\gamma=1$. 


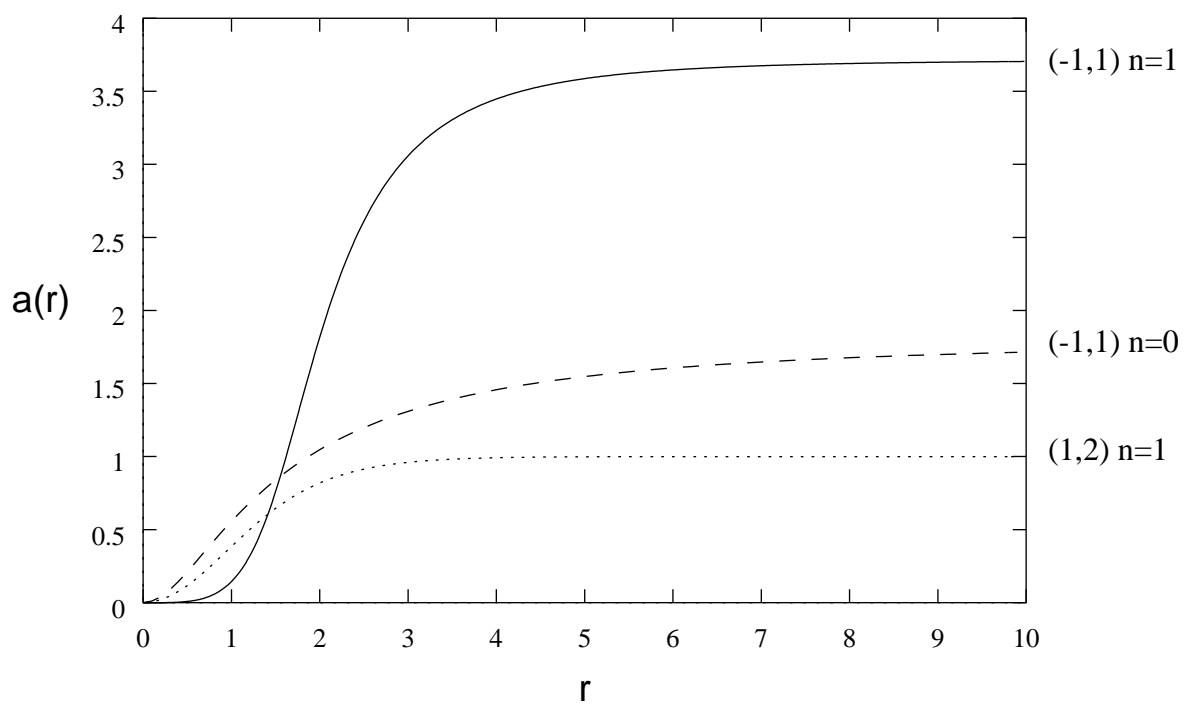

Fig. 3 Shapes of $a(r)$.

The solid line denotes non-topological vortex, the dotted line topological vortex, and the dashed line non-topological soliton. $(-1,1)$ means $\kappa=-1$ and $\gamma=1$. 